\title{
Ultrasound for Critical Care Physicians: Two's a Crowd
}

A 43 year old previously healthy woman was transferred to our hospital with refractory hypoxemia secondary to acute respiratory distress syndrome (ARDS) due to H1N1 influenza. She had presented to the outside hospital one week prior with cough and fevers. Chest radiography and computerized tomography of the chest revealed bilateral airspace opacities due to dependent consolidation and bilateral ground glass opacities. A transthoracic echocardiogram at the time of the patient's admission was reported as not revealing any significant abnormalities.

At the outside hospital she was placed on mechanical ventilation with low tidal volume, high Positive end-expiratory pressure $(20 \mathrm{~cm} \mathrm{H} 20)$, and a Fraction of inspired Oxygen (FiO2) of 1.0. Paralysis was later employed without significant improvement.

Upon arrival to our hospital, patient was severely hypoxemic with partial pressure of oxygen / FiO2 (P/F) ratio of 43. She was paralyzed with cis-atracurium and placed on airway pressure release ventilation (APRV) with the following settings (pressure high 28 $\mathrm{cm} \mathrm{H} 2 \mathrm{O}$, pressure low $0 \mathrm{~cm} \mathrm{H} 2 \mathrm{O}$, time high $5.5 \mathrm{sec}$, time low $0.5 \mathrm{sec}$ ). The patient remained severely hypoxemic with on oxygen saturation in the high 70 percent range.

A bedside echocardiogram was performed (Figures 1 and 2).

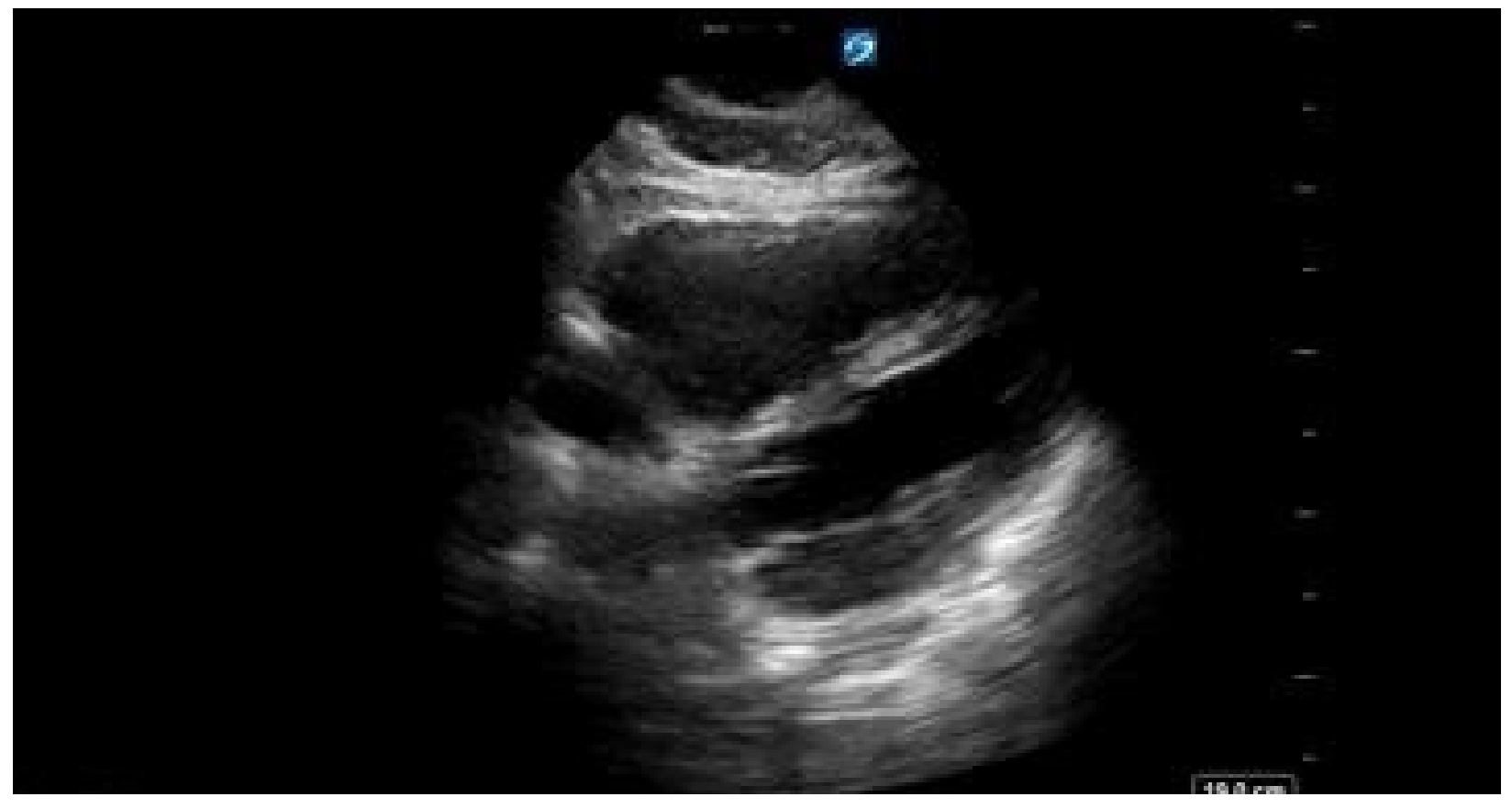

Figure 1. Subcostal long axis echocardiogram. 


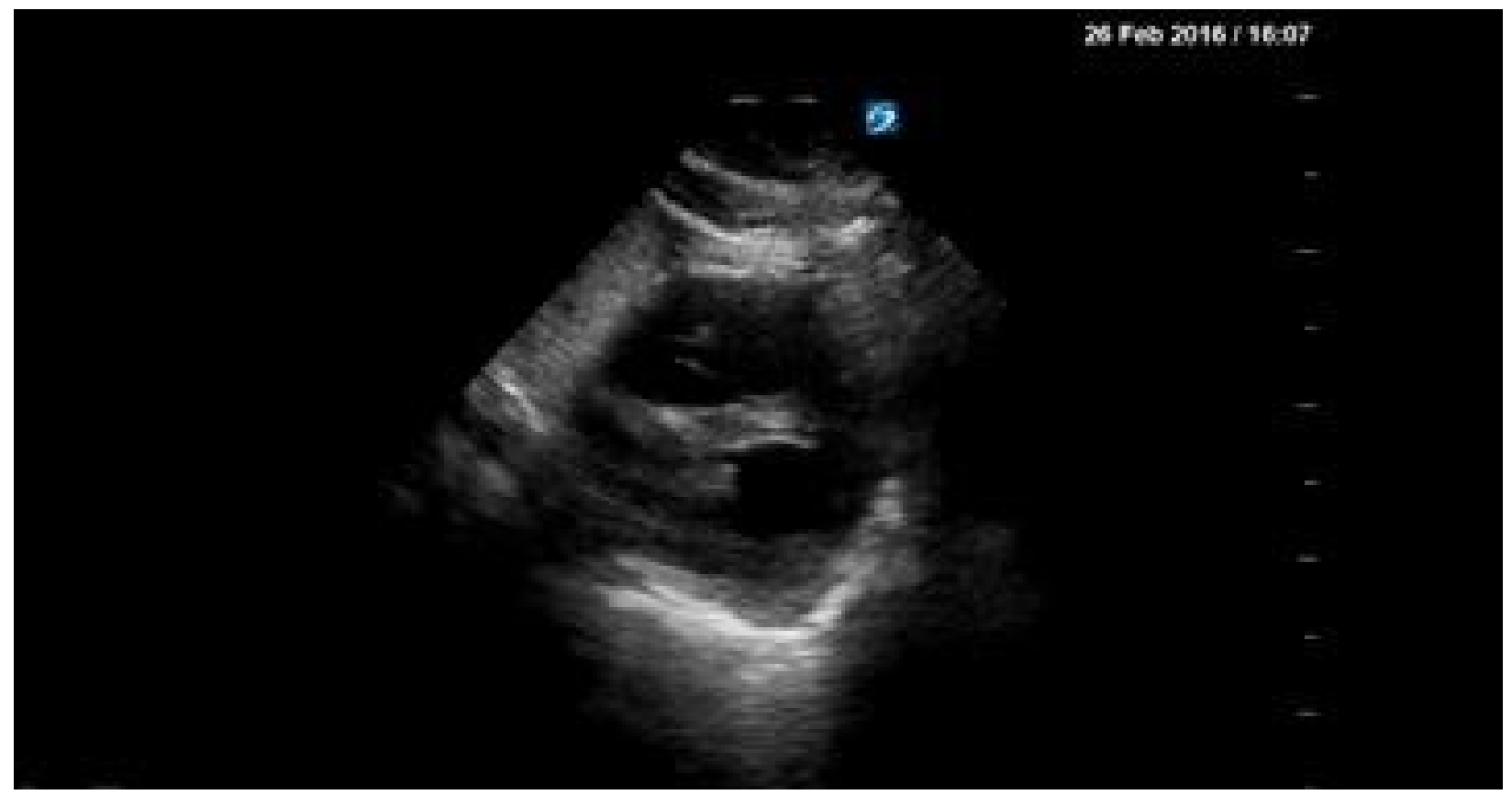

Figure 2. Subcostal short axis echocardiogram

What abnormality is demonstrated by the short and long axis subcostal views?

1. Acute cor pulmonale likely due to ARDS

2. Acute myocardial infarction

3. Low left-sided ejection fraction

4. Pericardial tamponade 


\section{Correct! \\ 1. Acute cor pulmonale likely due to ARDS}

Bedside echocardiography revealed hyperdynamic left ventricular function and signs of volume and pressure overload of the RV; increased RV end-diastolic volume on subcostal four chamber view and septal flattening "D sign" on subcostal short axis view respectively, Figures 3-4.

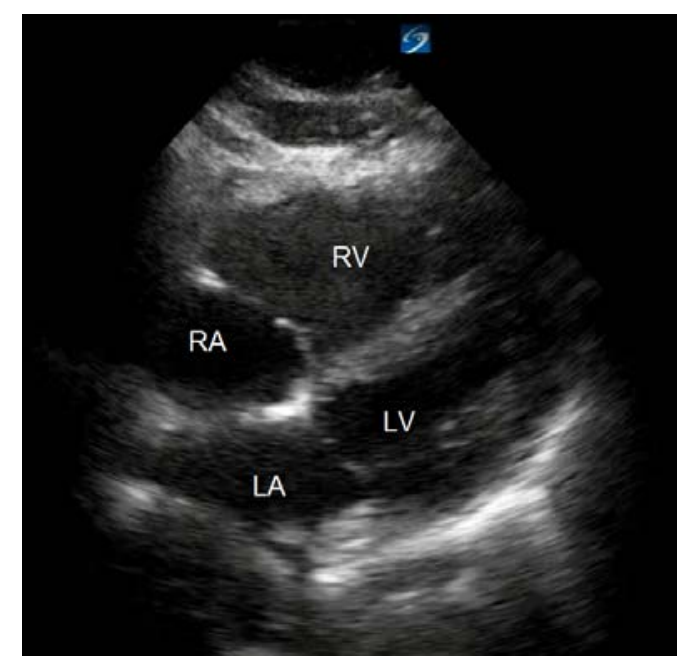

Figure 3. Still image of Figure 1/Video 1, demonstrating enlargement of the right ventricle (RV) compared to the left ventricle (LV). The RV being as large or larger than the $L V$ is a sign of abnormal dilation. The right atrium (RA) and left atrium (LA) are also labeled.

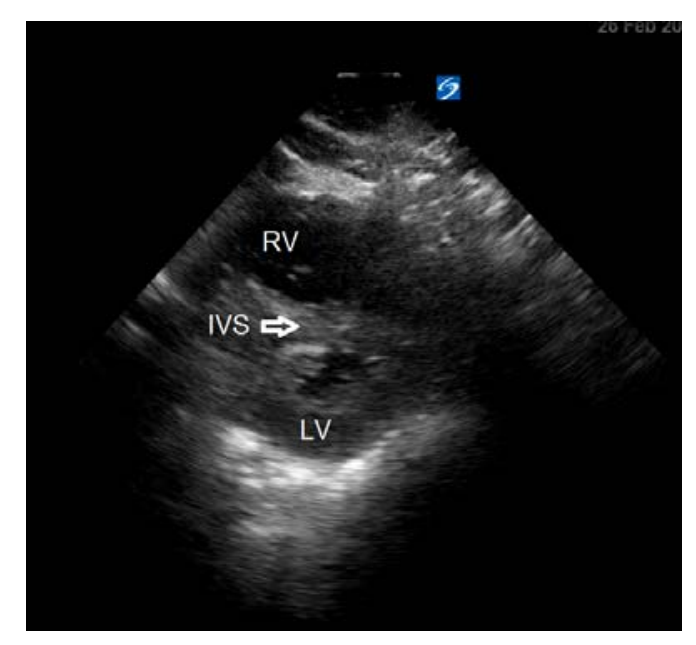

Figure 4. Still image of Figure 2/Video 2. In the subcostal short axis view, the RV is dilated and superior, whereas the LV is small with septal flattening. The normal short axis LV is round or shaped like an "O". In this case, the intra ventricular septum (labeled -IVS) has become flat and the LV is the shape of a "D" with the flat edge being the IVS. 
Given persistent hypoxemia and evidence of RV failure, in the setting of ARDS, acute Cor Pulmonale related to ARDS was diagnosed. It should be noted the patient had a negative CT angiogram. The patient was diuresed and placed on inhaled NO to reduce $\mathrm{RV}$ preload and reduce RV afterload, respectively. While this resulted in temporary improvement in hypoxemia, the patient worsened the next day and the patient was placed on veno-venous extra-corporeal membrane oxygenator therapy (ECMO). Despite improved understanding of pathophysiology of ARDS, it still carries significant mortality. It has long been known that ARDS causes, in addition to alveolar dysfunction, pulmonary capillary dysfunction leading to acute pulmonary hypertension. It is estimated that acute cor pulmonale(ACP) complicates $20-25 \%$ of ventilated patients with ARDS (1). Recent evidence suggests that "RV protective mechanical ventilation" may results in improved outcomes in patients with ARDS and ACP $(1,2)$.

In a review by Repesse et al. (1), the authors suggest routinely performing echocardiography-assisted analysis of RV function in ARDS given high incidence of RV failure in this patient population. They go on to describe an RV-protective ventilation strategy that includes strict limitation of plateau pressure $(<27 \mathrm{~cm} \mathrm{H} 2 \mathrm{O})$, driving pressure $(<17 \mathrm{~cm} \mathrm{H2O})$, diminution of PEEP and control of hypercapnia $(<60 \mathrm{mmHg})$, which they argue, may also be achieved with prone ventilation.

Jawad Abukhalaf MD and Michel Boivin MD

Division of Pulmonary, Critical Care and Sleep Medicine, University of New Mexico School of Medicine Albuquerque, NM USA

\section{References}

1. Repessé X, Charron C, Vieillard-Baron A. Acute cor pulmonale in ARDS: rationale for protecting the right ventricle. Chest. 2015 Jan;147(1):259-65. [CrossRef] [PubMed]

2. Vieillard-Baron A, Price LC, Matthay MA. Acute cor pulmonale in ARDS. Intensive Care Med. 2013 Oct;39(10):1836-8. [CrossRef] [PubMed] 